\title{
Race/Ethnicity, Dietary Acid Load, and Risk of End-Stage Renal Disease among US Adults with Chronic Kidney Disease
}

\author{
Deidra C. Crews ${ }^{a-c}$ Tanushree Banerjee ${ }^{d}$ Donald E. Wesson ${ }^{e}$ Hal Morgenstern ${ }^{f}$ \\ Rajiv Saran ${ }^{\text {g, h }}$ Nilka Ríos Burrows ${ }^{i}$ Desmond E. Williams ${ }^{j} \quad N^{2}$ il R. Powe ${ }^{d, k}$ \\ The Centers for Disease Control and Prevention Chronic Kidney Disease \\ Surveillance Team \\ a Division of Nephrology, Department of Medicine, Johns Hopkins University School of Medicine, Baltimore, MD, USA; \\ ${ }^{b}$ Welch Center for Prevention, Epidemiology and Clinical Research, Johns Hopkins Medical Institutions, Baltimore, \\ MD, USA; ' Johns Hopkins Center for Health Equity, Johns Hopkins Medical Institutions, Baltimore, MD, USA; dDivision \\ of General Internal Medicine, Department of Medicine, University of California, San Francisco, CA, USA; ${ }^{\circ}$ Diabetes \\ Health and Wellness Institute, Baylor Scott and White Health, Dallas, TX, USA; fDepartments of Epidemiology and \\ Environmental Health Sciences, School of Public Health, and Department of Urology, Medical School, University of \\ Michigan, Ann Arbor, MI, USA; ${ }^{9}$ Kidney Epidemiology and Cost Center, University of Michigan, Ann Arbor, MI, USA; \\ h Division of Nephrology, Department of Medicine, University of Michigan, Ann Arbor, MI, USA; 'Division of Diabetes \\ Translation, Centers for Disease Control and Prevention, Atlanta, GA, USA; ${ }^{j}$ Center for Global Health, Centers for \\ Disease Control and Prevention, Atlanta, GA, USA; ${ }^{k}$ Department of Medicine, San Francisco General Hospital, San \\ Francisco, CA, USA
}

\section{Keywords}

Acid-base · Epidemiology · Incidence

\begin{abstract}
Background: Dietary acid load (DAL) contributes to the risk of CKD and CKD progression. We sought to determine the relation of DAL to racial/ethnic differences in the risk of endstage renal disease (ESRD) among persons with CKD. Methods: Among 1,123 non-Hispanic black (NHB) and non-Hispanic white (NHW) National Health and Nutrition Examination Survey III participants with estimated glomerular filtration rate $15-59 \mathrm{~mL} / \mathrm{min} / 1.73 \mathrm{~m}^{2}$, DAL was estimated using the Remer and Manz net acid excretion $\left(\mathrm{NAE}_{\mathrm{es}}\right)$ formula and 24-h dietary recall. ESRD events were ascertained via
\end{abstract}

\section{KARGER}

(c) 2018 S. Karger AG, Basel

E-Mail karger@karger.com

www.karger.com/ajn linkage with Medicare. A competing risk model (accounting for death) was used to estimate the hazard ratio (HR) for treated ESRD, comparing NHBs with NHWs, adjusting for demographic, clinical and nutritional factors (body surface area, total caloric intake, serum bicarbonate, protein intake), and $\mathrm{NAE}_{\mathrm{es}}$. Additionally, whether the relation of $\mathrm{NAE}_{\mathrm{es}}$ with ESRD risk varied by race/ethnicity was tested. Results: At baseline, NHBs had greater $\mathrm{NAE}_{\mathrm{es}}$ (50.9 vs. $44.2 \mathrm{mEq} /$ day) than NHWs. It was found that $22 \%$ developed ESRD over a median of 7.5 years. The unadjusted HR comparing NHBs to NHWs was 3.35 (95\% Cl 2.51-4.48) and adjusted HR (for factors above) was 1.68 (95\% Cl 1.18-2.38). A stronger association of NAE with risk of ESRD was observed among NHBs (adjusted HR per $\mathrm{mEq} /$ day increase in NAE 1.21, 95\% Cl 1.121.31 ) than that among NHWs (HR 1.08, 95\% Cl 0.96-1.20), $p$ 
interaction for race/ethnicity $\times \mathrm{NAE}_{\mathrm{es}}=0.004$. Conclusions: Among US adults with CKD, the association of DAL with progression to ESRD is stronger among NHBs than NHWs. DAL is worthy of further investigation for its contribution to kidney outcomes across race/ethnic groups.

(C) 2018 S. Karger AG, Basel

\section{Introduction}

Blacks experience more rapid decline in kidney function than whites $[1,2]$ and have a greater risk of end-stage renal disease (ESRD) $[3,4]$. While the contributions of apolipoprotein L1 (APOL1) risk variants to racial disparities in chronic kidney disease (CKD) have received attention in the recent past $[1,5]$, focus on the potential role of modifiable risk factors, such as diet, in these disparities is also warranted [6].

High dietary acid load (DAL) is associated with adverse kidney outcomes [7-9]; interventional studies have shown that alkaline diets (e.g., rich in fruits and vegetables) provide benefits to the kidney [10-12]. Explanatory mechanisms underlying the association of DAL and kidney injury may include tubular toxicity of elevated ammonium concentration [13], increased endothelin and aldosterone [14-16], and increased angiotensin II activity [17], with the latter agents yielding the physiologic benefit of increased distal nephron acidification but with the attendant pathophysiologic consequence of long-term kidney injury.

We reported in a nationally representative sample that non-Hispanic black (NHB) race was associated with higher DAL than non-Hispanic white (NHW) race [9] and high DAL was associated with increased risk of ESRD among US adults with CKD [8]. However, the relationship of racial differences in DAL to racial disparities in CKD progression is unknown. Understanding these relationships could inform interventions designed to mitigate disparities in ESRD risk.

The objective of the present study was to determine the contribution of racial differences in DAL, quantified by dietary net acid excretion ( $\mathrm{NAE}_{\mathrm{es}}$ ), to the more rapid progression to ESRD observed among NHBs as compared to NHWs $[3,4]$. We also sought to determine whether the association of DAL and risk of ESRD varied by race. Our study was conducted among a nationally representative sample of US adults with CKD stages 3 or 4 [18] using data from the National Health and Nutrition Examination Survey (NHANES) III, with follow-up for ESRD outcomes.

Race/Ethnicity, DAL, and Risk of ESRD among US Adults with CKD

\section{Methods}

Study Population and Baseline Data

NHANES III was a national probability sample of 34,955 US non-institutionalized civilians conducted between 1988 and 1994 by the Centers for Disease Control and Prevention's National Center for Health Statistics (NCHS). For this analysis, we included NHB and NHW participants $\geq 20$ years of age $(n=14,223)$ who did not have missing data on dietary intake $(n=12,279)$, had an estimated glomerular filtration rate (eGFR) $\geq 15$ and $<60 \mathrm{~mL} / \mathrm{min}$ per $1.73 \mathrm{~m}^{2}(n=1,261)$, and were not pregnant (final $n=1,123$ ). NHANES III participants provided informed consent.

\section{Sociodemographic and Clinical Measurements}

Medical history and demographic data were collected through a standardized survey conducted at participants' homes followed by a medical examination and laboratory testing that occurred in the mobile examination center [19]. Sociodemographic factors were assessed during the interview. Racial/ethnic categories were self-reported by participants. Self-reported information on socioeconomic position (education and income) was obtained during the interview portions of the survey. Income was assessed using the poverty income ratio, a ratio of household income to the household poverty level [19]. Diabetes was defined by self-report or measured hemoglobin A1c $\geq 6.5 \%$ [20]. Hypertension was defined by self-report, a measured average systolic blood pressure $\geq 140 \mathrm{~mm}$ $\mathrm{Hg}$ or average diastolic blood pressure $\geq 90 \mathrm{~mm} \mathrm{Hg}$, or reported use of antihypertensive medication [21].

\section{Measurement and Classification of Serum Bicarbonate and \\ Kidney Parameters}

Serum bicarbonate was measured by the phosphoenolpyruvate method using the Hitachi 737 multichannel analyzer (Boehringer Mannheim Diagnostics, Indianapolis, IN, USA). Serum creatinine measurements that were obtained using a kinetic rate Jaffé method in NHANES III were recalibrated to standardized creatinine measurements obtained at the Cleveland Clinic Research Laboratory (Cleveland, OH, USA) as standard creatinine $=0.184+0.9603$ NHANES III-measured serum creatinine [22]. Random spot urine samples were obtained and frozen. Urine albumin was measured using a solid-phase fluorescence immunoassay, and urine creatinine was measured using the modified Jaffé kinetic method in the same laboratory. Estimated GFR was calculated using the isotope dilution mass spectrometry traceable 4 -variable Modification of Diet in Renal Disease (MDRD) Study equation for calibrated creatinine [23]. Albuminuria, which was determined by the urinary albumin-to-creatinine ratio, was expressed as milligrams of albumin per gram of creatinine using American Diabetes Association categories: normal ( $<30 \mathrm{mg} / \mathrm{g})$ and albuminuria ( $\geq 30 \mathrm{mg} / \mathrm{g})$ [24].

Dietary Assessment and DAL

Dietary intake data collected in NHANES III were used to estimate the types and amounts of foods and beverages consumed during the 24 -h period before the interview (midnight to midnight) and estimate the intake of energy and nutrients from those foods and beverages. The non-bicarbonate anions (protein and phosphorus) intake and the mineral cations (potassium, magnesium, and calcium) intake of foods by participants were derived from the dietary intake data. Potential renal acid load (PRAL) of foods reported by the participants was calculated using the model 
by Remer and Manz, (PRAL [mEq/day] $=0.493$ protein $[\mathrm{g}]+$ 0.0373 phosphorus [mg] 20.0213 potassium [mg] 20.0263 magnesium [mg] 20.01253 calcium [mg]) [25]. DAL was estimated as $\mathrm{NAE}_{\mathrm{es}}(\mathrm{mEq} /$ day $)=\mathrm{PRAL}+$ organic acids $(\mathrm{OAs})$, where OA was calculated as OA $(\mathrm{mEq} /$ day $)=\left(\mathrm{BSA}\left[\mathrm{m}^{2}\right] \times 41[\mathrm{mEq} /\right.$ day per 1.73 $\left.\mathrm{m}^{2}\right] / 1.73 \mathrm{~m}^{2}$ ) [25], as used in our previous work $[8,9,26]$.

\section{Outcome Ascertainment}

Our primary outcome of interest was progression to ESRD. In NHANES III, ESRD was defined as the initiation of chronic dialysis. ESRD events and mortality follow-up data from the time of the survey (1988-1994) through December 31, 2006, were determined from the Medicare ESRD Registry and National Death Index, which were linked to NHANES III data [27]. ESRD data are available for those NHANES respondents who agreed to provide personal identification data to NCHS and for whom NCHS was able to match with US Renal Data System administrative records.

\section{Statistical Analyses}

Baseline characteristics of study participants stratified by NHB and NHW race/ethnicity were compared using chi-square tests for categorical variables. For continuous variables, we checked normality assumptions and used one-way analysis of variance for normally distributed variables. In cases where the assumptions were not met, we used the Kruskal-Wallis test for continuous variables. We investigated the association of race with the development of ESRD while accounting for the competing risk of death prior to ESRD using the method by Fine and Gray [28]. We estimated the hazard ratio (HR) of ESRD with linear and quadratic terms for $\mathrm{NAE}_{\text {es }}$ in our multivariable Cox regression models to assess the relation between DAL and the rate of ESRD among race groups. We assessed the proportionality of each categorical covariate by plotting $\log (-\log [$ survival] $)$ versus $\log$ of survival time and survival function versus survival time. We tested the proportionality of continuous variables using Schoenfeld residual plots and examined their statistical significance. Product terms were used to examine possible effect modification by race/ethnicity (race/ethnicity $\times \mathrm{NAE}_{\mathrm{es}}$ ). All analyses included the NHANES survey sample weights to account for the complex sample design of the survey, and we followed the analytical guidelines for NHANES III data as proposed by the Centers for Disease Control and Prevention [29]. Results were considered statistically significant if $p<0.05$. All analyses were performed using SAS 9.2 (SAS Institute, Inc., Cary, NC, USA).

\section{Sensitivity Analysis}

We conducted sensitivity analyses in which we defined CKD stages 3 and 4 using eGFR calculated from the creatinine-based CKD-EPI equation [30] and repeated our primary analyses.

\section{Results}

\section{Participant Characteristics}

A total of 1,123 NHB and NHW NHANES III participants with an eGFR between 15 and $59 \mathrm{~mL} / \mathrm{min} / 1.73 \mathrm{~m}^{2}$ were included in our study. There were no major differences in the sociodemographic and clinical characteris- tics of the participants who were included and those who were excluded, except age - those included had a mean age of 69.5 compared to 75.4 years for those excluded.

NHBs and NHWs differed on several characteristics examined (Table 1). NHBs were younger, more likely to live in poverty, less likely to have completed more than high school and more likely to have diabetes and/or hypertension than were NHWs. Compared with NHWs, NHBs had a greater baseline prevalence of albuminuria and a slightly lower median eGFR. The median estimated DAL, calculated as $\mathrm{NAE}_{\mathrm{es}}$, was $46.3 \mathrm{mEq} /$ day (interquartile range [IQR] 34.6-58.4 mEq/day) for the full sample, and NHBs had higher PRAL and $\mathrm{NAE}_{\mathrm{es}}$ than NHWs.

\section{Associations of Race and DAL with Risk of ESRD}

A total of 248 (22.1\%) participants developed ESRD over a median of 7.5 (IQR 4.5-12.4) years of follow-up, including 154 (43.9\%) NHBs and 94 (12.2\%) NHWs. In unadjusted Cox regression models accounting for the competing risk of death, NHBs had greater risk of ESRD than NHWs (HR 3.35, 95\% CI 2.51-4.48; Table 2). Subsequent models adjusting for covariates yielded an attenuated association of NHB race with risk of ESRD, with a HR of $1.68,95 \%$ CI 1.18-2.38 in our final model, which was adjusted for socio-demographic (age, sex, and poverty income ratio), clinical (diabetes, hypertension, eGFR and albumin-to-creatinine ratio), and nutritional (body surface area, total caloric intake, serum bicarbonate, protein intake, and $\mathrm{NAE}_{\mathrm{es}}$ ) factors. Effect modification by race/ethnicity $\times \mathrm{NAE}_{\text {es }}$ was observed, with a $p$ value of 0.004 for the interaction in our final model. We proceeded by fitting separate models for NHBs and NHWs (Table 3). The estimated adjusted HR of ESRD for each $1 \mathrm{mEq} /$ day increase in $\mathrm{NAE}_{\text {es }}$ was 1.21 (95\% CI 1.12-1.31) among NHBs and 1.08 (95\% CI 0.96-1.20) among NHWs.

\section{Sensitivity Analyses}

When we defined CKD stages 3 and 4 using GFR estimated from the CKD Epidemiology Collaboration (CKDEPI) equation ( $n=991$; Table 4$)$, results were similar to those of our models using the MDRD Study equation. In unadjusted Cox regression models accounting for the competing risk of death, NHBs had greater risk of ESRD than NHWs (HR 2.48, 95\% CI 1.81-3.39). In our fully adjusted model, the HR was $1.91,95 \%$ CI $1.28-2.84$, and effect modification by race/ethnicity $\times \mathrm{NAE}_{\text {es }}$ was observed ( $p$ interaction 0.03 ). In race-/ethnicity-specific models, the adjusted $\mathrm{HR}$ for ESRD associated with $\mathrm{NAE}_{\mathrm{es}}$ was 1.28 (95\% CI 1.17-1.40) among NHBs and 1.10 (95\% CI 0.90-1.28) among NHWs. 
Table 1. Baseline characteristics of NHANES III participants with CKD stages 3 or 4, overall and by race/ethnicity

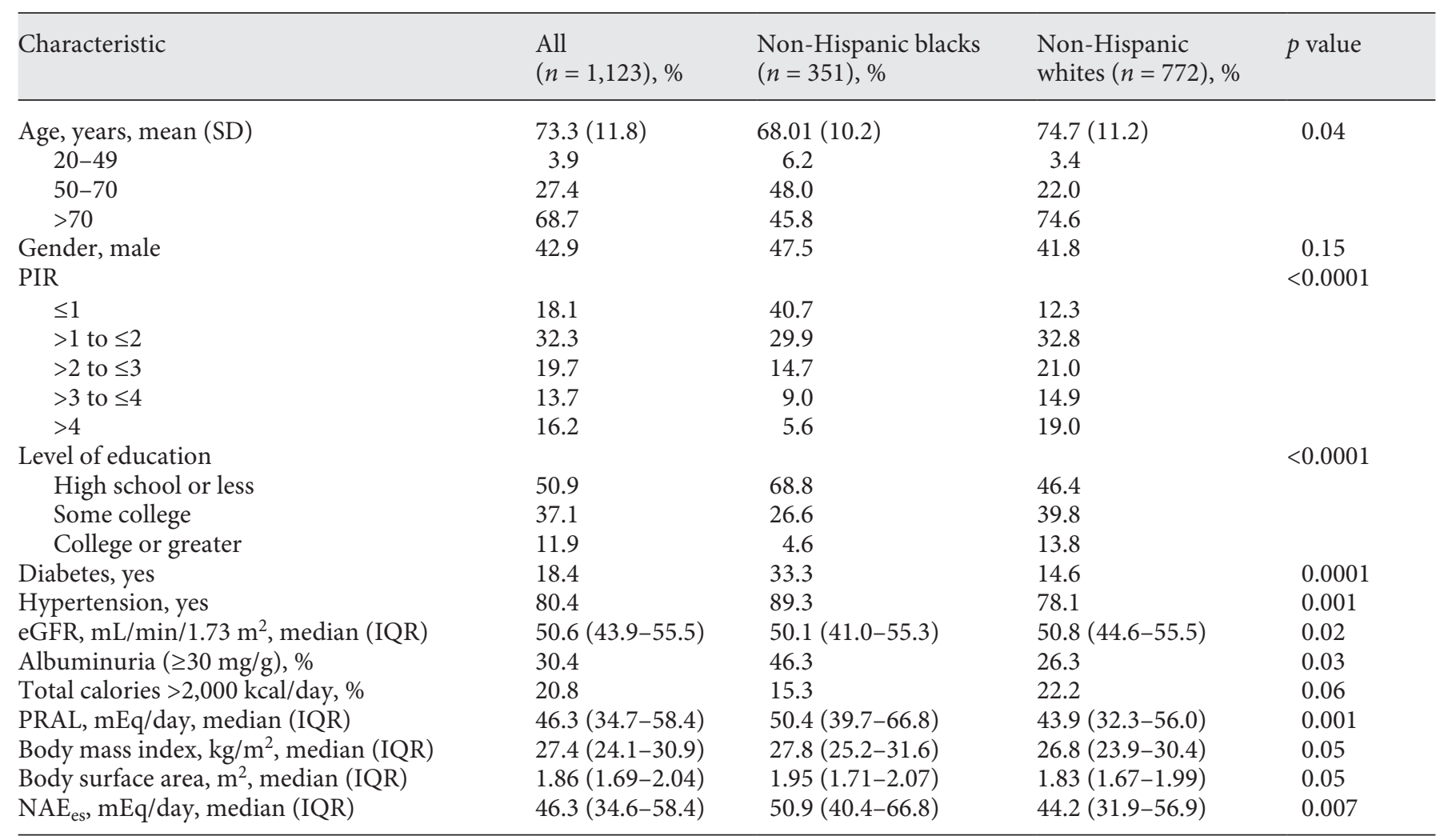

CKD defined using MDRD study equation for eGFR.

PIR is the ratio of family income to federal poverty threshold. Hypertension was defined by self-report, average systolic blood pressure $\geq 140$ or average diastolic blood pressure $\geq 90 \mathrm{~mm} \mathrm{Hg}$, or use of medications. Diabetes was defined by self-report or measured hemoglobin A1c $\geq 6.5 \%$.

NHANES, National Health and Nutrition Examination Survey; CKD, chronic kidney disease; eGFR, estimated glomerular filtration rate; $\mathrm{IQR}$, interquartile range; $\mathrm{PIR}$, poverty income ratio; $\mathrm{PRAL}$, potential renal acid load; $\mathrm{NAE}_{\mathrm{es}}$, net acid excretion; MDRD, modification of diet in renal disease.

Table 2. HRs for CKD progression to ESRD comparing non-Hispanic blacks to non-Hispanic whites in the NHANES III

\begin{tabular}{lll}
\hline Models & Baseline variables included & $\begin{array}{l}\text { HR (95\% CI) for ESRD accounting } \\
\text { for the competing risk of death } \\
(n=1,123, \text { ESRD events }=248)\end{array}$ \\
\hline 1 & Non-Hispanic blacks versus non-Hispanic whites, unadjusted $^{*}$ & $3.35(2.51-4.48)$ \\
2 & Model 1 + NAE $^{*}$ & $3.21(2.40-4.30)$ \\
3 & Model 2 + age, gender & $3.25(2.41-4.39)$ \\
4 & Model 3 + PIR & $2.62(1.92-3.58)$ \\
5 & Model 4 + body surface area, total caloric intake, serum bicarbonate, protein intake & $3.52(2.55-4.85)$ \\
6 & Model 5 + diabetes, hypertension & $2.64(1.90-3.65)$ \\
7 & Model 6 + eGFR, urinary ACR & $1.68(1.18-2.38)$
\end{tabular}

* Model includes both linear and quadratic terms for $\mathrm{NAE}_{\mathrm{es}}$.

HR, hazard ratio; CKD, chronic kidney disease; ESRD, end stage renal disease; NHANES, National Health and Nutrition Examination Survey; eGFR, estimated glomerular filtration rate; PIR, poverty income ratio; $\mathrm{NAE}_{\mathrm{es}}$, net acid excretion; ACR, albumin-to-creatinine ratio. 
Table 3. Race/ethnicity stratified HRs for the association of $\mathrm{NAE}_{\mathrm{es}}$ with risk of CKD progression to ESRD in the NHNES III

\begin{tabular}{|c|c|c|}
\hline $\mathrm{NAE}_{\mathrm{es}} *$ (unadjusted) & $1.31(1.20-1.44)$ & $1.02(0.97-1.06)$ \\
\hline+ Age, gender & $1.26(1.16-1.38)$ & $1.03(0.98-1.08)$ \\
\hline + Diabetes, hypertension & $1.17(1.09-1.26)$ & $1.04(0.97-1.12)$ \\
\hline + eGFR, urinary ACR & $1.21(1.12-1.31)$ & $1.08(0.96-1.20)$ \\
\hline
\end{tabular}

* Model includes both linear and quadratic terms for net acid excretion.

HRs, hazard ratios; $\mathrm{NAE}_{\mathrm{es}}$, net acid excretion; CKD, chronic kidney disease; ESRD, end stage renal disease; NHANES, National Health and Nutrition Examination Survey; PIR, poverty income ratio; eGFR, estimated glomerular filtration rate; ACR, albumin-tocreatinine ratio.

Table 4. Baseline characteristics of NHANES III participants with CKD stages 3 or 4 (defined using CKD-EPI equation), overall and by race/ethnicity

\begin{tabular}{|c|c|c|c|c|}
\hline Characteristic & $\begin{array}{l}\text { All } \\
(n=991), \%\end{array}$ & $\begin{array}{l}\text { Non-Hispanic } \\
\text { blacks }(n=309), \%\end{array}$ & $\begin{array}{l}\text { Non-Hispanic } \\
\text { whites }(n=682), \%\end{array}$ & $p$ value \\
\hline Age, years, mean (SD) & $73.2(11.8)$ & $67.7(12.7)$ & $74.5(11.2)$ & 0.03 \\
\hline $20-50$ & 4.0 & 6.7 & 3.3 & \\
\hline $50-70$ & 27.5 & 48.8 & 22.2 & \\
\hline$>70$ & 68.5 & 44.5 & 74.5 & \\
\hline Gender, male & 42.9 & 46.3 & 42.0 & 0.27 \\
\hline$\leq 1$ & 17.1 & 39.6 & 11.5 & \\
\hline $1-2$ & 32.2 & 29.3 & 32.9 & \\
\hline $2-3$ & 19.8 & 15.2 & 20.9 & \\
\hline $3-4$ & 14.0 & 9.8 & 15.0 & \\
\hline$>4$ & 16.9 & 6.1 & 19.6 & \\
\hline Education & & & & $<0.0001$ \\
\hline Hypertension, yes & 80.4 & 88.4 & 78.5 & 0.003 \\
\hline $\mathrm{eGFR}, \mathrm{mL} / \mathrm{min} / 1.73 \mathrm{~m}^{2}$, median (IQR) & $47.3(41.6-55.5)$ & $50.2(41.1-55.2)$ & $50.8(44.5-55.6)$ & 0.04 \\
\hline Albuminuria $(>30 \mathrm{mg} / \mathrm{g})$ & 29.9 & 45.1 & 26.1 & $<0.0001$ \\
\hline Total calories $>2,000 \mathrm{kcal} /$ day & 21.7 & 16.5 & 23.1 & 0.09 \\
\hline $\mathrm{NAE}_{\mathrm{es}}, \mathrm{mEq} /$ day, median (IQR) & $47.3(41.6-53.6)$ & $47.4(41.2-52.9)$ & $47.2(41.7-53.8)$ & 0.67 \\
\hline
\end{tabular}

* CKD-EPI equation was used in sensitivity analysis to define chronic kidney disease, given its reported greater precision over the MDRD study equations [51]. PIR is the ratio of family income to poverty threshold. Hypertension was defined by self-report, average systolic blood pressure $>140$ or average diastolic blood pressure $>90 \mathrm{~mm} \mathrm{Hg}$, or use of medication.

NHANES, National Health and Nutrition Examination Survey; CKD, chronic kidney disease; CKD-EPI, CKD epidemiology collaboration; PIR, poverty income ratio; eGFR, estimated glomerular filtration rate; IQR, interquartile range; $\mathrm{NAE}_{\mathrm{es}}$, net acid excretion; MDRD, modification of diet in renal disease. 


\section{Discussion}

Among a nationally representative sample of $\mathrm{NHB}$ and NHW US adults with CKD, we found that NHBs had greater DAL than NHWs. DAL contributed little to explaining NHBs greater risk of progression to ESRD beyond traditional risk factors, although the association of DAL with the risk of ESRD was stronger among NHBs than among NHWs. Our findings were robust to adjustment for multiple potential confounders of the associations of race/ethnicity, diet, and CKD progression, including albuminuria and total caloric intake, and to the definition of CKD (using MDRD versus CKD-EPI).

This is among the first studies to examine the role of diet in racial disparities in CKD progression. Our work advances the findings of prior studies documenting poorer diet quality among blacks, including their lower likelihood of following a Dietary Approaches to Stop Hypertension trial-accordant diet than whites [31,32], although it has been shown that blacks have the potential to derive the greatest blood-pressure-lowering benefit from the diet [33]. The Dietary Approaches to Stop Hypertension diet, which is high in fruits and vegetables, moderate in low-fat dairy products, and low in animal protein, but with substantial amounts of plant protein from legumes and nuts [34], is low in DAL [35] and is associated with lower risk of CKD [32] and kidney function decline [36]. Lower rates of blacks, as compared to whites, following diets low in DAL may be, in part, due to their greater socioeconomic barriers to healthful eating. For example, in 2011, 35\% of African Americans lived below the US federal poverty threshold (USD 22,350 annually for a family of 4), compared to $13 \%$ of whites [37]. Other potential reasons for poorer diet quality among African Americans compared to whites include African Americans' perceptions of healthful dietary practices, cultural and familial norms, and preferences [38-40].

Our finding of a stronger association between DAL and risk of ESRD among NHBs could be due to the unaccounted differences between NHBs and NHWs with CKD in the United States - including cause of CKD. In our study, NHBs with CKD were about 7 years younger, were more likely to have diabetes and/or hypertension, and were substantially more likely to have albuminuria than NHWs. It is therefore very likely that the causes of CKD differed between the 2 race groups. We would expect, for example, given the estimated prevalence of high risk APOL1 alleles among persons with CKD who are African American (19\%) [5], that a substantial proportion of our NHB study population had high-risk APOL1 alleles and therefore a greater prevalence of focal segmental glomerulosclerosis [41] than NHWs. In a study examining the potential modifiers of the association of high-risk APOL1 variants with CKD progression among African Americans with hypertension-attributed kidney disease, high-risk $A P O L 1$ variant status was associated with a 2.3 times greater hazard of CKD progression among individuals with lower DAL (as estimated by net endogenous acid production). In contrast, among individuals with higher DAL, high-risk APOL1 variant status was minimally associated with CKD progression (HR 1.41, 95\% CI 0.97-2.07) [42], suggesting that in the setting of poor diet quality, CKD progression is less attributable to this genetic risk factor. Based upon this study and ours, the relation of dietary factors to the APOL1-associated risk of CKD progression among African Americans may warrant further study.

Another potential explanation for our findings is related to the potential effects of DAL on blood pressure. Based upon data from animal models [15, 43, 44], high DAL is postulated to lead to increases in angiotensin II, endothelin-1, and aldosterone, which can ultimately lead to blood pressure elevation as well as kidney fibrosis. NHBs with CKD and hypertension have poorer blood pressure control than NHWs with the same conditions [45], which in part, might be mediated by dietary factors, among other factors.

The limitations of our study warrant consideration. First, error in measuring diet in NHANES could have biased our findings, particularly if it varied by race/ethnicity. Second, we lacked longitudinal measures of dietary patterns and other potential time-varying mediators of the association of diet with CKD progression, including blood pressure and glycemic control. Third, we lacked several measures that may have differentially influenced NHBs and NHWs risk of progression to ESRD. These include constitutional factors such as the aforementioned genetic risk factors, as well as social determinants of health closely linked to diet and/or race that were not examined in our study, such as residential segregation [46] and discrimination [47]. Fourth, we were unable to account for differences in rates of preemptive kidney transplantation, which is performed for relatively few US patients each year (2,192 patients in 2009) but at unequal rates across race/ethnicities [48]. We do not believe that this substantially influenced our results, however, as we observed similar racial differences in progression to ESRD (defined by dialysis initiation) as have been previously reported $[3,49,50]$. Fifth, our study only included NHBs and NHWs. Future studies with greater representation of multiple race/ethnicity groups might further ex- 
amine this question. The strengths of our study included its longitudinal design; the examination of a potentially modifiable contributor to racial disparities in CKD progression and a potential pathophysiologic mechanism to account for DAL and progression to ESRD; and the consideration of mortality during follow-up.

Dietary approaches may warrant further consideration as therapeutic targets for CKD patients, including those at high risk for progression. Together with other interventions, such as blood pressure control, DAL may be an important target for addressing the greater risk of $\mathrm{CKD}$ progression among NHBs. Evidence pointing to the potential benefits of healthful diets in slowing CKD progression is mounting; however, many individuals face barriers to following such dietary patterns, including food insecurity [51] and a lack of healthful foods in neighborhood stores [46]. Thus, dietary interventions targeting populations at high risk of CKD progression, such as
NHBs, might better serve these populations if contextual factors posing challenges to making lifestyle modifications are addressed [38].

\section{Disclosure Statement}

The authors report no conflicts of interest relative to this manuscript.

\section{Acknowledgments}

This work was supported by contract NU58DP006255-01-00 of the Centers for Disease Control and Prevention. The findings and conclusions in this report are those of the authors and do not necessarily represent the official position of the Centers for Disease Control and Prevention. Dr. Deidra C. Crews was supported by the National Institute of Diabetes and Digestive and Kidney Diseases, National Institutes of Health Grant K23-DK097184.

\section{References}

1 Peralta CA, Bibbins-Domingo K, Vittinghoff E, Lin F, Fornage M, Kopp JB, Winkler CA: APOL1 genotype and race differences in incident albuminuria and renal function decline. J Am Soc Nephrol 2016;27:887-893.

2 Peralta CA, Vittinghoff E, Bansal N, Jacobs D Jr, Muntner P, Kestenbaum B, Lewis C, Siscovick D, Kramer H, Shlipak M, et al: Trajectories of kidney function decline in young black and white adults with preserved GFR: results from the Coronary Artery Risk Development in Young Adults (CARDIA) study. Am J Kidney Dis 2013;62:261-266.

3 McClellan WM, Warnock DG, Judd S, Muntner P, Kewalramani R, Cushman M, McClure LA, Newsome BB, Howard G: Albuminuria and racial disparities in the risk for ESRD. J Am Soc Nephrol 2011;22:1721-1728.

4 System USRD: Annual Data Report.

5 Parsa A, Kao WH, Xie D, Astor BC, Li M, Hsu CY, Feldman HI, Parekh RS, Kusek JW, Greene TH, et al: APOL1 risk variants, race, and progression of chronic kidney disease. $\mathrm{N}$ Engl J Med 2013;369:2183-2196.

6 Crews DC, Pfaff T, Powe NR: Socioeconomic factors and racial disparities in kidney disease outcomes. Semin Nephrol 2013;33:468-475.

7 Scialla JJ, Appel LJ, Astor BC, Miller ER 3rd, Beddhu S, Woodward M, Parekh RS, Anderson CA; African American Study of Kidney Disease and Hypertension Study Group: Net endogenous acid production is associated with a faster decline in GFR in African Americans. Kidney Int 2012;82:106-112.

8 Banerjee T, Crews DC, Wesson DE, Tilea AM, Saran R, Rios-Burrows N, Williams DE, Powe NR; Centers for Disease Contro and Preven- tion Chronic Kidney Disease Surveillance Team: High dietary acid load predicts ESRD among adults with CKD. J Am Soc Nephrol 2015;26:1693-1700.

9 Banerjee T, Crews DC, Wesson DE, Tilea A, Saran R, Rios Burrows N, Williams DE, Powe NR; Centers for Disease Control and Prevention Chronic Kidney Disease Surveillance Team: Dietary acid load and chronic kidney disease among adults in the United States. BMC Nephrol 2014;15:137.

10 Goraya N, Simoni J, Jo C, Wesson DE: Dietary acid reduction with fruits and vegetables or bicarbonate attenuates kidney injury in patients with a moderately reduced glomerular filtration rate due to hypertensive nephropathy. Kidney Int 2012;81:86-93.

11 Goraya N, Simoni J, Jo CH, Wesson DE: Treatment of metabolic acidosis in patients with stage 3 chronic kidney disease with fruits and vegetables or oral bicarbonate reduces urine angiotensinogen and preserves glomerular filtration rate. Kidney Int 2014;86:10311038.

12 Goraya N, Simoni J, Jo CH, Wesson DE: A comparison of treating metabolic acidosis in CKD stage 4 hypertensive kidney disease with fruits and vegetables or sodium bicarbonate. Clin J Am Soc Nephrol 2013;8:371-381.

13 Nath KA, Hostetter MK, Hostetter TH: Increased ammoniagenesis as a determinant of progressive renal injury. Am J Kidney Dis 1991;17:654-657.

14 Phisitkul S, Khanna A, Simoni J, Broglio K, Sheather S, Rajab MH, Wesson DE: Amelioration of metabolic acidosis in patients with low GFR reduced kidney endothelin produc- tion and kidney injury, and better preserved GFR. Kidney Int 2010;77:617-623.

15 Wesson DE, Simoni J: Acid retention during kidney failure induces endothelin and aldosterone production which lead to progressive GFR decline, a situation ameliorated by alkali diet. Kidney Int 2010;78:1128-1135.

16 Wesson DE, Simoni J, Broglio K, Sheather S: Acid retention accompanies reduced GFR in humans and increases plasma levels of endothelin and aldosterone. Am J Physiol Renal Physiol 2011;300:F830-F837.

17 Wesson DE, Jo CH, Simoni J: Angiotensin IImediated GFR decline in subtotal nephrectomy is due to acid retention associated with reduced GFR. Nephrol Dial Transplant 2015; 30:762-770.

18 KDOQI Clinical Practice Guidelines for chronic kidney disease: evaluation, classification and stratification (http://www2.kidney. org/professionals/KDOQI/guidelines_ckd/ toc.htm).

19 Plan and operation of the Third National Health and Nutrition Examination Survey, 1988-94. Series 1: programs and collection procedures. Vital Health Stat 1994;32:1-407.

20 International Expert Committee: International Expert Committee report on the role of the $\mathrm{A} 1 \mathrm{C}$ assay in the diagnosis of diabetes. Diabetes Care 2009;32:1327-1334.

21 Chobanian AV, Bakris GL, Black HR, Cushman WC, Green LA, Izzo JL Jr, Jones DW, Materson BJ, Oparil S, Wright JT Jr, et al: The seventh report of the joint national committee on prevention, detection, evaluation, and treatment of high blood pressure: the JNC 7 report. JAMA 2003;289:2560-2572. 
22 Coresh J, Selvin E, Stevens LA, Manzi J, Kusek JW, Eggers P, Van Lente F, Levey AS: Prevalence of chronic kidney disease in the United States. JAMA 2007;298:2038-2047.

23 Levey AS, Coresh J, Greene T, Marsh J, Stevens LA, Kusek JW, Van Lente F; Chronic Kidney Disease Epidemiology Collaboration: Expressing the modification of diet in renal disease study equation for estimating glomerular filtration rate with standardized serum creatinine values. Clin Chem 2007;53:766772 .

24 Molitch ME, DeFronzo RA, Franz MJ, Keane WF, Mogensen CE, Parving HH, Steffes MW; American Diabetes Association: Nephropathy in diabetes. Diabetes Care 2004;27(suppl 1):S79-S83.

25 Remer T, Manz F: Potential renal acid load of foods and its influence on urine $\mathrm{pH}$. J Am Diet Assoc 1995;95:791-797.

26 Rebholz CM, Coresh J, Grams ME, Steffen LM, Anderson CA, Appel LJ, Crews DC: Dietary acid load and incident chronic kidney disease: results from the ARIC study. Am J Nephrol 2015;42:427-435.

27 National Center for Health Statistics: The Third National Health and Nutrition Examination Survey (NHANES III) Linked Mortality File: Matching Methodology: In Hyattsville, MD, 2005.

28 Fine JP, Gray RJ: A proportional hazards model for the subdistribution of a competing risk. J Am Stat Assoc 1999;94:496-509.

29 National Center for Health Statistics: Analytical and Reporting Guidelines: The Third National Health and Nutrition Examination Survey, 1988-1994. In Hyattsville, MD, 1996.

30 Levey AS, Stevens LA, Schmid CH, Zhang YL, Castro AF 3rd, Feldman HI, Kusek JW, Eggers P, Van Lente F, Greene T, et al: A new equation to estimate glomerular filtration rate. Ann Intern Med 2009;150:604-612.

31 Mellen PB, Gao SK, Vitolins MZ, Goff DC Jr: Deteriorating dietary habits among adults with hypertension: DASH dietary accordance, NHANES 1988-1994 and 1999-2004. Arch Intern Med 2008;168:308-314.

32 Crews DC, Kuczmarski MF, Miller ER 3rd, Zonderman AB, Evans MK, Powe NR: Dietary habits, poverty, and chronic kidney dis- ease in an urban population. J Renal Nutr 2015;25:103-110.

33 Svetkey LP, Simons-Morton D, Vollmer WM, Appel LJ, Conlin PR, Ryan DH, Ard J, Kennedy BM: Effects of dietary patterns on blood pressure: subgroup analysis of the Dietary Approaches to Stop Hypertension (DASH) randomized clinical trial. Arch Intern Med 1999;159:285-293.

34 Appel LJ, Moore TJ, Obarzanek E, Vollmer WM, Svetkey LP, Sacks FM, Bray GA, Vogt TM, Cutler JA, Windhauser MM, et al: A clinical trial of the effects of dietary patterns on blood pressure. DASH Collaborative Research Group. N Engl J Med 1997;336:1117-1124.

35 Scialla JJ, Anderson CA: Dietary acid load: a novel nutritional target in chronic kidney disease? Adv Chronic Kidney Dis 2013;20:141149.

36 Lin J, Hu FB, Curhan GC: Associations of diet with albuminuria and kidney function decline. Clin J Am Soc Nephrol 2010;5:836-843.

37 DeNavas-Walt C, Proctor BD, Smith JC: U.S Census Bureau, Current Population Reports, P60-243, Income, Poverty, and Health Insurance Coverage in the United States. 2011. In, Washington, 2012.

38 Johnson AE, Boulware LE, Anderson CA, Chit-ua-aree T, Kahan K, Boyer LL, Liu Y, Crews DC: Perceived barriers and facilitators of using dietary modification for CKD prevention among African Americans of low socioeconomic status: a qualitative study. BMC Nephrol 2014;15:194.

39 Airhihenbuwa CO, Kumanyika S, Agurs TD, Lowe A, Saunders D, Morssink CB: Cultural aspects of African American eating patterns. Ethn Health 1996;1:245-260.

40 Tirodkar MA, Jain A: Food messages on African American television shows. Am J Public Health 2003;93:439-441.

41 Genovese G, Friedman DJ, Ross MD, Lecordier L, Uzureau P, Freedman BI, Bowden DW, Langefeld CD, Oleksyk TK, Uscinski Knob AL, et al: Association of trypanolytic ApoL1 variants with kidney disease in African Americans. Science 2010;329:841-845.

42 Chen TK, Choi MJ, Kao WH, Astor BC, Scialla JJ, Appel LJ, Li L, Lipkowitz MS, Wolf M, Parekh RS, et al: Examination of potential modifiers of the association of APOL1 alleles with CKD progression. Clin J Am Soc Nephrol 2015;10:2128-2135.

43 Wesson DE, Jo CH, Simoni J: Angiotensin II receptors mediate increased distal nephron acidification caused by acid retention. Kidney Int 2012;82:1184-1194.

44 Phisitkul S, Hacker C, Simoni J, Tran RM, Wesson DE: Dietary protein causes a decline in the glomerular filtration rate of the remnant kidney mediated by metabolic acidosis and endothelin receptors. Kidney Int 2008; 73 192-199.

45 Plantinga LC, Miller ER 3rd, Stevens LA, Saran R, Messer K, Flowers N, Geiss L, Powe NR; Centers for Disease Control and Prevention Chronic Kidney Disease Surveillance Team: Blood pressure control among persons without and with chronic kidney disease: US trends and risk factors 1999-2006. Hypertension 2009;54:47-56.

46 Franco M, Diez Roux AV, Glass TA, Caballero B, Brancati FL: Neighborhood characteristics and availability of healthy foods in Baltimore. Am J Prev Med 2008;35:561-567.

47 Beydoun MA, Poggi-Burke A, Zonderman $A B$, Rostant OS, Evans MK, Crews DC: Perceived discrimination and longitudinal change in kidney function among Urban adults. Psychosom Med 2017;79:824-834.

48 Grams ME, Massie AB, Coresh J, Segev DL: Trends in the timing of pre-emptive kidney transplantation. J Am Soc Nephrol 2011;22: $1615-1620$

49 Whittle JC, Whelton PK, Seidler AJ, Klag MJ: Does racial variation in risk factors explain black-white differences in the incidence of hypertensive end-stage renal disease? Arch Intern Med 1991;151:1359-1364.

50 Hsu CY, Lin F, VittinghoffE, Shlipak MG: Racial differences in the progression from chronic renal insufficiency to end-stage renal disease in the United States. J Am Soc Nephrol 2003;14:2902-2907.

51 Crews DC, Kuczmarski MF, Grubbs V, Hedgeman E, Shahinian VB, Evans MK, Zonderman AB, Burrows NR, Williams DE, Saran R, Powe NR; Centers for Disease Control and Prevention Chronic Kidney Disease Surveillance Team: Effect of food insecurity on chronic kidney disease in lower-income Americans. Am J Nephrol 2014;39:27-35. 